\title{
Scheduling and Coding for Unicast in Multi-Radio Systems: the Two-User Case * (Invited Paper)
}

\author{
Feng Xue \\ Corporate Technology Group, Intel Corporation \\ feng.xue1@intel.com
}

\begin{abstract}
Cross-layer scheduling and coding for the coexistence between two wireless networks, a primary network and a secondary network, are considered for the case of two users. Both networks want to transmit to the users, which, however, cannot receive simultaneously on the two radios. Subject to fading and random interruption from the primary network modeled as continuous Markov chain, the secondary network optimizes its scheduling (probing/transmission time /ACK) and coding to maximize its throughput. Performances of several algorithms are considered, which characterize the relationship among important system parameters and shed light on system design.
\end{abstract}

\section{Categories and Subject Descriptors}

C.2.1 [Network Architecture and Design]: Wireless communication; H.1 [Models and Principles]: General

\section{General Terms \\ Performance}

\section{Keywords}

Wireless communication, cross-layer design, network coding, scheduling

\section{INTRODUCTION}

Recently, advanced technology has allowed a single wireless device to have multiple radios. For example, WiFi and WWAN technology have been combined in laptop computer (e.g. Intel WiMAX/WiFi Link 5350) or cell phone (e.g., Nokia 6310 GSM/WiFi phone). In the near future there will be many devices with two or more radios, providing ubiquitous connectivity and various new services. Typically the radios on one device can not work simultaneously due to several reasons. One reason is that they might work on

*WICON'08, November 17-19, 2008, Maui, Hawaii, USA. Copyright 2008 ICST 978-963-9799-36-3

\author{
Xue Yang \\ Corporate Technology Group, Intel Corporation \\ xue.yang@intel.com
}

the same or similar frequency bands, and interference (leaking in the latter case) would deteriorate the performance. Another reason is that the radios may share certain processing circuitry due to cost considerations. As the density of devices grows, coordination and scheduling among devices with multi-radios become a challenging problem. IEEE has initiated effort towards the co-existence of different networks/standards $[1,2]$.

There has been research on co-existence of multi-radios. Coexistence of nodes, each with a different radio, has been investigated. For example, the coexistence of Ultra-Wideband (UWB) and narrow-band cellular systems was studied in [3]. [4] presented a physical-layer based analysis of the coexistence issues of UWB with other WiFi devices in the same spectrum. In [5], a link-layer protocol was proposed for a single node to coordinate the operation of multiple wireless network cards tuned to non-overlapping frequency channels. Cognitive radio, where devices can sense environment and utilize idle frequency bands more efficiently, has attracted a lot research interests [6]. In [7], both lower and upper bounds were considered for multi-radio mesh networks based on a confliction based model. In [8], how to pro-actively choose channel was investigated based on a Markov chain model.

Network coding has also been studied for dense wireless environment. It takes advantage of the broadcast nature of wireless communications, and allows users to help each other. In [9], a cross-layer design shows that nodes can forward partial information based on corrupted PHY packets towards later hops so that by combining several of them, correct decoding can be made. In [10], broadcast by using Markov Decision Process was studied. In [11], broadcast stability region in random access through network coding was characterized. Yet network coding requires extra overhead in terms of indexing and combining, and it is not clear whether network coding is always better [12].

The co-existence issue of multi-radios in a dense environment is a cross-layer problem. In a traditional single-radio network, media access control is designed for sharing the wireless media efficiently among devices which use the same mechanism. One example is WiFi networks which uses CSMA /CA for media access. In a multi-radio network, when to access and how to back up on one radio should also depend on the activity of the other radios. Different radios/standards have different physical layer coding, and, with the wide spreading of smart antenna technology, a node has multi- 
ple PHY-layer coding modes (diversity, beamforming, multiplexing, etc.) to choose from. Combined with node density and traffic pattern, designing the optimal coexistence certainly involves all the factors.

In this paper, we model the transmission among nodes with two radios to consider both physical layer channel fading and MAC layer scheduling. Analytical and numerical results on the performance of several coding and scheduling strategies are provided. With a modeling from a practice point of view, the purpose is to understand how important parameters such as MAC activity, PHY-layer fading, transmission period and probing/ACK period influence the performance.

To be more specific, we consider the influence of fading and activity in the primary network to the secondary network. Focusing on the simple two-user case, analysis shows that 1)PHY-layer coding needs to consider the fading process; 2) Transmission period should be carefully calibrated to maximize the throughput; and 3) Simple coding encounters too much lost due to interruptions from the primary network when the latter is busy. We also show that network coding improves the performance, however advanced coding which takes care of the corrupted pieces would greatly help. Our study justifies the need for cross-layer joint scheduling and coding optimization in multi-radio environment.

The rest of the paper is structured as follows. In Section II, network model and physical layer fading model are presented. In Section III, performance of several simple scheduling strategies are presented. In Section IV, performances of simple network coding is considered, and limit cases are presented in Section V. Section VI presents numerical results and Section VII concludes with comments and future work.

\section{MODELING COEXISTENCE AND FAD- ING}

We assume that there are two nodes each having two radios, one for network Net 1 and the other for network Net2. Downlink transmissions are considered as shown in Figure 1. That is, both Net 1 and Net2 are trying to send messages to both nodes. A node cannot listen to both networks at the same time. Net2 is the primary network, meaning it schedules its transmission without considering Net1. Whenever Net2 needs to transmit to a node, the node stops its current receiving in Net1, if any, and starts listening in Net2.

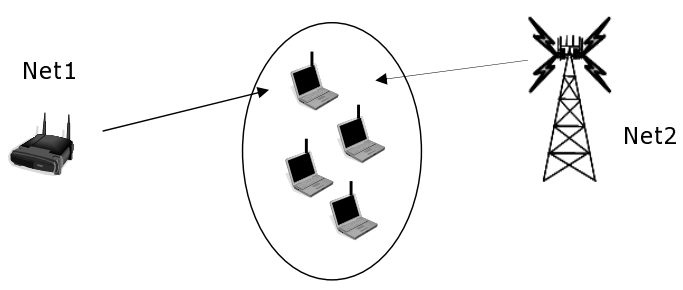

Figure 1: System

We model the traffic in Net2 as a continuous time Markov birth-death process. Each user's activity in Net2 is independent of the other. If user $k$ is served by Net2, the service period has an exponential distribution with parameter $\lambda_{2}$.
The idle period of user $k$ from Net2 has an exponential distribution with parameter $\lambda_{1}$. Note that user $\mathrm{k}$ could be served by Net1 in this period.

When Net1 transmits to a user, we assume that it can transmit $W$ complex symbols per second with power $\mathrm{P}$. The corresponding received symbol can be modeled as $y=h x+n$, where $h$ is the complex channel gain and $n$ is an independent regular Gaussian noise with unit variance. According to information theory, the channel can achieve $W \log \left(1+|h|^{2} P\right)$ bits per second when $h$ is constant. In practice, however, $h$ changes with time due to fading. Within the channel coherence time, the channel can be considered constant. The channel $h$ changes to other realizations when time is longer than the channel coherence time. Block fading channel model has been consider to model this phenomenon (e.g. [14]). In this paper we use the following continuous Rayleigh Markov chain model. For each channel realization, the channel keeps constant for a period with exponential distribution of parameter $\lambda$. After this period the channel changes to independent realization chosen based on Rayleigh distribution. That is $\operatorname{Pr}\left(|h|^{2}>a\right)=e^{-a}$.

We assume that $N e t 1$ fixes transmission time for each packet as $T_{s}$. Before each transmission, Net 1 takes $T_{p}$ seconds to set up the transmission and probe the channel quality. After each transmission, Net 1 takes $T_{a}$ seconds to end the transmission and get acknowledgement. To simplify the modeling, we assume that probing and acknowledgement will not be influenced by Net2 or bad channel. Denote $T_{a}+T_{p}=\mu T_{s}$, with $\mu$ being typically less than one. For each strategy discussed later, more details about probing and acknowledgement will be specified.

To model the physical layer channel coding/decoding, we take a outage formulation. Each packet is encoded according to certain rate $R=\log \left(1+\left|h_{0}\right|^{2} P\right)$ for $T_{s}$ seconds. A receiver can successfully decode the packet only if during the whole transmission the channel gain is always such that $|h|^{2} \geq$ $\left|h_{0}\right|^{2}$. In the sequel, denote

$$
W_{0}:=W \log \left(1+\left|h_{0}\right|^{2} P\right)
$$

as the information bit rate per symbol achieved by a successful decoding, and call $\left|h_{0}\right|^{2}$ the coding threshold.

Given the above modeling, we know there are two events that can prevent a node from receiving from Net1 successfully: 1) When Net1 is transmitting but the node is serviced by Net2; and 2) When Net1 is transmitting and the node is listening in Net1, but the channel gain is small.

We have the following observation.

Lemma 1. Suppose that Net1 codes the channel code according to threshold $\left|h_{0}\right|^{2}$. Then the time that the fading channel to a node is above this threshold has an exponential distribution with parameter $\left(1-e^{-\left|h_{0}\right|^{2}}\right) \lambda$, and the time that the channel is below the threshold has also an exponential distribution with parameter $e^{-\left|h_{0}\right|^{2}} \lambda$.

Proof: Given any time $T>0$ and that the current channel state is above the threshold, the number of times that the 
fading will update the channel during $T$ seconds is a Poisson random variable with mean $T \lambda$. So the probability that there are $k$ updates is $\frac{(T \lambda)^{k}}{k !} e^{-T \lambda}$. The probability that the channel is always good during the $T$ seconds is

$\sum_{k=0}^{\infty} \frac{(T \lambda)^{k}}{k !} e^{-T \lambda} \operatorname{Pr}\left(|h|^{2} \geq\left|h_{0}\right|^{2}\right)^{k}=e^{p_{0} T \lambda-T \lambda}=e^{-\left(1-p_{0}\right) T \lambda}$

where $p_{0}=\operatorname{Pr}\left(|h|^{2} \geq\left|h_{0}\right|^{2}\right)=e^{-\left|h_{0}\right|^{2}}$. So the good state lasts according to an exponential distribution with parameter $\left(1-p_{0}\right) \lambda$. Similarly, the bad channel distributes according to $\exp \left(p_{0} \lambda\right)$.

\section{SIMPLE CODING WITH SCHEDULING}

In this section, several scheduling algorithms are considered.

We first consider the basic round robin scheduling without considering the existence of Net2, which serves as the basis for comparison.

\subsection{Round Robin without Considering Co-Existence of Net2}

In this scheduling, Net1 serves each node alternatively with $T_{s}+T_{p}+T_{a}=T_{s}+\mu T_{s}$ seconds, as if there is no Net2. This models the case when Net1 does not know the existence of another interfering radio and assumes full control of the access time.

Assume that Net1 sets the channel coding threshold as $\left|h_{0}\right|^{2}$. Because Net2's activity is described by a continuous Markov chain and Net1's channel fading is described by an independent stationary process, the combined process is stationary. At the start of Node A's serving periods (of length $T_{s}+\mu T_{s}$ ), the probability that Node A has a good PHY channel and is available from Net2 is $\frac{1 / \lambda_{1}}{1 / \lambda_{1}+1 / \lambda_{2}} e^{-\left|h_{0}\right|^{2}}$, when the process converges to its stationary distribution.

Given that Node A starts a serving period $T_{s}$ with good PHY channel and idle Net2, the probability that it will not be interrupted by Net2 or meet a bad channel during the next $T_{s}$ seconds is $e^{-\left(\lambda_{1}+\left(1-p_{0}\right) \lambda\right) T_{s}}$, with $p_{0}:=e^{-\left|h_{0}\right|^{2}}$. So the information throughput for Node A is

$$
\frac{1}{2} \frac{\frac{1 / \lambda_{1}}{1 / \lambda_{1}+1 / \lambda_{2}} e^{-\left|h_{0}\right|^{2}} \cdot W_{0} T_{s} \cdot e^{-\left(\lambda_{1}+(1-p) \lambda\right) T_{s}}}{T_{s}+\mu T_{s}} .
$$

The sum rate for both nodes $\mathrm{A}$ and $\mathrm{B}$ is thus

$$
\begin{aligned}
& \frac{\frac{1 / \lambda_{1}}{1 / \lambda_{1}+1 / \lambda_{2}} e^{-\left|h_{0}\right|^{2}} \cdot W_{0} \cdot e^{-\left(\lambda_{1}+(1-p) \lambda\right) T_{s}}}{1+\mu} \\
& =\frac{W}{1+\mu} \frac{\left(1 / \lambda_{1}\right) e^{-\lambda_{1} T_{s}}}{1 / \lambda_{1}+1 / \lambda_{2}} \\
& \quad \cdot \log \left(1+\left|h_{0}\right|^{2} P\right) e^{-\left|h_{0}\right|^{2}} e^{-\left(1-e^{-\left|h_{0}\right|^{2}}\right) \lambda T_{s}} .
\end{aligned}
$$

We have the following result on the best threshold $\left|h_{0}\right|^{2}$.

TheOrem 1. The best threshold $\left|h_{0}\right|^{2}$ for given $T_{s}, \mu, \lambda$, $\lambda_{1}, \lambda_{2}$ is the unique solution of the following equation:

$$
\frac{P}{(1+x P) \log (1+x P)}=1+e^{-x} \lambda T_{s} .
$$

Proof: See Appendix A.

Note that $\lambda_{1}, \lambda_{2}$ are not in the optimization. Also note that $e^{-\left|h_{0}\right|^{2}} \log \left(1+\left|h_{0}\right|^{2} P\right)$ is the expected throughput if the fading does not change (flat fading). In this case, it is easy to show that the best threshold $\left|h_{0}\right|^{2}$ is such that $\frac{P}{(1+x P) \log (1+x P)}=1$. Compared to the optimal threshold taking care of the fading where $\frac{P}{(1+x P) \log (1+x P)}=1+e^{-x} \lambda T_{s}$, this shows that a smaller and conservative $\left|h_{0}\right|^{2}$ needs to be chosen to combat the fading. It also shows that one should use almost the same threshold if $e^{-\left|h_{0}\right|^{2}} \lambda T_{s}$ is small, which is true if the original threshold is large or $\lambda T_{s}$ is small. Recall that $\lambda T_{s}$ is the average number of channel changes.

\subsection{Transmit Whenever Net2 Is Idle}

In this scheduling, Net1 detects each node sequentially, and sends to a node for $T_{s}$ seconds whenever it is idle from Net2 at the end of probing. Note that each detection with the scheduling at the end of the transmission cost $T_{p}+T_{a}=\mu T_{s}$ seconds.

Assume that the coding threshold is $\left|h_{0}\right|^{2}$. According to the previous analysis, the threshold is set to be the solution to

$$
\frac{P}{(1+x P) \log (1+x P)}=1+e^{-x} \lambda T_{s} .
$$

Since the transmission of Net1 does not depend on the fading, the process is described by Net2's activity. For each user, denote Net2's status as ' 0 ' is Net2 is transmitting to it, and ' 1 ' if not. Then for each user, Net2 is a continuous Markov chain with transition matrix

$$
H:=\left[h_{11}(t), h_{10}(t) ; h_{01}(t), h_{00}(t)\right],
$$

with

$$
\begin{aligned}
& h_{11}(t)=\frac{\lambda_{1}}{\lambda_{1}+\lambda_{2}} e^{-\left(\lambda_{1}+\lambda_{2}\right) t}+\frac{\lambda_{2}}{\lambda_{1}+\lambda_{2}}, \\
& h_{00}(t)=\frac{\lambda_{2}}{\lambda_{1}+\lambda_{2}} e^{-\left(\lambda_{1}+\lambda_{2}\right) t}+\frac{\lambda_{1}}{\lambda_{1}+\lambda_{2}} .
\end{aligned}
$$

This can be found in standard text such as [15].

For the two user case, we construct the following discrete Markov chain. The states are $\left(B_{1} B_{2}\right)$, where $B_{1}$ denotes the "current" serving node's status after the probe $\left(T_{p}\right)$. That is, $B_{1}=1$ means that the node Net1 is probing is not busy in Net2; $B_{1}=0$ means otherwise. $B_{2}$ is the status of the other node when it was probed by Net1 the last time. We thus get a chain with 4 states: $\{00,01,10,11\}$. (Note that a transmission consists three parts $T_{p}, T_{s}$ and $T_{a}$.)

We have $\operatorname{Pr}(00 \mid 00)=h_{00}\left(2 \mu T_{s}\right)$, where $h_{00}\left(2 \mu T_{s}\right)$ is the probability that the other node is busy after $2 \mu T_{s}$ seconds since the last probe (which was busy too in Net2). Similarly, we have

$\begin{array}{ll}\operatorname{Pr}(10 \mid 00)=h_{01}\left(2 \mu T_{s}\right), & \operatorname{Pr}(00 \mid 00)=h_{00}\left(2 \mu T_{s}\right) \\ \operatorname{Pr}(10 \mid 01)=h_{11}\left((1+2 \mu) T_{s}\right), & \operatorname{Pr}(00 \mid 01)=h_{10}\left((1+2 \mu) T_{s}\right) \\ \operatorname{Pr}(01 \mid 10)=h_{00}\left((1+2 \mu) T_{s}\right), & \operatorname{Pr}(11 \mid 10)=h_{01}\left((1+2 \mu) T_{s}\right) \\ \operatorname{Pr}(11 \mid 11)=h_{11}\left((2+2 \mu) T_{s}\right), & \operatorname{Pr}(01 \mid 11)=h_{10}\left((2+2 \mu) T_{s}\right) \\ \text { Denote } a:=\operatorname{Pr}(10 \mid 00), b:=\operatorname{Pr}(00 \mid 01), c:=\operatorname{Pr}(11 \mid 10) \text { and } \\ d:=\operatorname{Pr}(01 \mid 11), \text { and denote }\left(\pi_{00}, \pi_{01}, \pi_{10}, \pi_{11}\right) \text { the stationary }\end{array}$ 
distribution. We have

$$
\begin{aligned}
& \pi_{00} \bar{a}+\pi_{01} b=\pi_{00} \\
& \pi_{11} d+\pi_{10} \bar{c}=\pi_{01} \\
& \pi_{01} \bar{b}+\pi_{00} a=\pi_{10} \\
& \pi_{11} \bar{d}+\pi_{10} c=\pi_{11} .
\end{aligned}
$$

Solving the equations we get $\pi_{01}=(a / b) \pi_{00}, \pi_{11}=\frac{a / b}{d / c} \pi_{00}$ and $\pi_{10}=\frac{a}{b} \pi_{00}$. Thus we have

$$
\frac{\pi_{10}+\pi_{11}}{\pi_{00}+\pi_{01}}=\frac{1+c / d}{1+b / a} .
$$

Consider the chain $\left(B_{1} B_{2}\right)$. A successful transmission can only happen when the state is 10 or 11 . Given that the current serving node is in state ' 1 ', the packet transmission of $T_{s}$ seconds is successful only if during the period the PHY channel is always better than $\left|h_{0}\right|^{2}$ and there is no interruption from Net2. This happens with probability $p_{0} e^{-\lambda\left(1-p_{0}\right) T_{s}} \cdot e^{-\lambda_{1} T_{s}}$ with $p_{0}:=e^{-\left|h_{0}\right|^{2}}$. Since each '1' state consumes $(1+\mu) T_{s}$ seconds, the overall information rate is thus

$$
\begin{aligned}
& \underline{\left(\pi_{10}+\pi_{11}\right) p_{0} \cdot e^{-\lambda\left(1-p_{0}\right) T_{s}} e^{-\lambda_{1} T_{s}} \cdot W_{0} T_{s}} \\
& \left(\pi_{10}+\pi_{11}\right)(1+\mu) T_{s}+\left(\pi_{00}+\pi_{01}\right) \mu T_{s} \\
& =\frac{\left(\pi_{10}+\pi_{11}\right) e^{-\left|h_{0}\right|^{2}} \cdot e^{-\lambda\left(1-e^{-\left|h_{0}\right|^{2}}\right) T_{s}} e^{-\lambda_{1} T_{s}}}{\left(\pi_{10}+\pi_{11}\right)(1+\mu)+\left(\pi_{00}+\pi_{01}\right) \mu} \\
& \text {. } W \log \left(1+\left|h_{0}\right|^{2} P\right) \\
& =\frac{e^{-\left|h_{0}\right|^{2}} \cdot e^{-\lambda\left(1-e^{-\left|h_{0}\right|^{2}}\right) T_{s}} e^{-\lambda_{1} T_{s}} \cdot W \log \left(1+\left|h_{0}\right|^{2} P\right)}{1+\frac{\lambda_{1}\left(1-e^{\left.-\left(\lambda_{1}+\lambda_{2}\right)(1+2 \mu) T_{s}\right)}\right.}{\lambda_{2}\left(1-e^{\left.-\left(\lambda_{1}+\lambda_{2}\right) 2 \mu T_{s}\right)}\right.}} \\
& (1+\mu)+\mu \frac{1+\frac{\lambda_{1}\left(1-e-\left(\lambda_{1}+\lambda_{2}\right)\left(1+e^{\left.-\left(\lambda_{1}+\lambda_{2}\right) 2 \mu T_{s}\right)}\right.\right.}{\left.\lambda_{2}(1-)_{s}\right)}}{1+\frac{\lambda_{2}\left(1-e^{\left.-\left(\lambda_{1}+\lambda_{2}\right)(1+2 \mu) T_{s}\right)}\right.}{\lambda_{1}\left(1-e^{\left.-\left(\lambda_{1}+\lambda_{2}\right) 2(1+\mu) T_{s}\right)}\right.}} \\
& =\frac{e^{-\left|h_{0}\right|^{2}} e^{-\lambda\left(1-e^{-\left|h_{0}\right|^{2}}\right) T_{s}} \log \left(1+\left|h_{0}\right|^{2} P\right) \cdot W e^{-\lambda_{1} T_{s}}}{(1+\mu)+\mu \frac{1+\frac{\lambda_{1}\left(1-e^{\left.-\left(\lambda_{1}+\lambda_{2}\right)(1+2 \mu) T_{s}\right)}\right.}{\lambda_{2}\left(1-e^{\left.-\left(\lambda_{1}+\lambda_{2}\right) 2 \mu T_{s}\right)}\right.}}{1+\frac{\lambda_{2}\left(1-e^{\left.-\left(\lambda_{1}+\lambda_{2}\right)(1+2 \mu) T_{s}\right)}\right.}{\lambda_{1}\left(1-e^{\left.-\left(\lambda_{1}+\lambda_{2}\right) 2(1+\mu) T_{s}\right)}\right.}}}
\end{aligned}
$$

\subsection{Transmit only When Both Net2 Is Idle and Channel Is Good}

We consider the following scheduling. Net1 alternatively detects each user to see whether it is idle in Net2 and has a good channel above the coding threshold. If so, Net1 transmits to this user for $T_{s}$ seconds, otherwise, it turns to the next user for detection.

This process can also be described by a Markov chain similarly as in the above subsection. However, here more states are needed for denoting the physical channel quality. The Markov chain can be constructed as follows. Let $\left(X_{1} X_{2}\right.$; $\left.Y_{1} Y_{2}\right)$ denote the status of the two nodes. $X_{1}$ and $X_{2}$ denote the current serving node's status immediately after the probing. $X_{1}$ denotes its Net1 channel status, with $X_{1}=1$ meaning that the node has a good PHY channel and $X_{1}=0$ otherwise. $X_{2}$ denotes the status of the node's status in Net2, with '1' being idle and '0' being busy. $Y_{1}$ and $Y_{2}$ denote the status of the node last probed, with $Y_{1}$ denoting the status of the channel in Net1 and $Y_{2}$ the status of Net2.
This Markov chain has 16 states. Because $Y_{1} Y_{2}$ denote the status of the node probed last time, each state can transit into four possible states. The probability of going from state $\left(a_{1} a_{2} a_{3} a_{4}\right)$ to state $\left(b_{1} b_{2} a_{1} a_{2}\right)$ is determined by $f_{11}(t)$ and $f_{00}(t)$ defined in (1) and the following transition functions of the fading channels for given $\left|h_{0}\right|^{2}$ :

$$
\begin{aligned}
& g_{11}(t):=\frac{\tilde{\lambda}_{1}}{\tilde{\lambda}_{1}+\tilde{\lambda}_{2}} e^{-\left(\tilde{\lambda}_{1}+\tilde{\lambda}_{2}\right) t}+\frac{\tilde{\lambda}_{2}}{\tilde{\lambda}_{1}+\tilde{\lambda}_{2}}, \\
& g_{00}(t):=\frac{\tilde{\lambda}_{2}}{\tilde{\lambda}_{1}+\tilde{\lambda}_{2}} e^{-\left(\tilde{\lambda}_{1}+\tilde{\lambda}_{2}\right) t}+\frac{\tilde{\lambda}_{1}}{\tilde{\lambda}_{1}+\tilde{\lambda}_{2}},
\end{aligned}
$$

where $\tilde{\lambda}_{1}:=\lambda\left(1-e^{-\left|h_{0}\right|^{2}}\right)$ and $\tilde{\lambda}_{2}:=\lambda e^{-\left|h_{0}\right|^{2}}$. To illustrate, the first 4 columns of the state transition matrix can be described as follows.

$$
\begin{aligned}
& \operatorname{Pr}(0000 \mid 0000)=\operatorname{Pr}(0001 \mid 0100)=\operatorname{Pr}(0010 \mid 1000) \\
& \quad=f_{00}\left(2 \mu T_{s}\right) g_{00}\left(2 \mu T_{s}\right), \\
& \operatorname{Pr}(0011 \mid 1100)=f_{00}\left((1+2 \mu) T_{s}\right) g_{00}\left((1+2 \mu) T_{s}\right) ; \\
& \operatorname{Pr}(0100 \mid 0000)=\operatorname{Pr}(0101 \mid 0100)=\operatorname{Pr}(0110 \mid 1000) \\
& \quad=f_{00}\left(2 \mu T_{s}\right) \bar{g}_{00}\left(2 \mu T_{s}\right), \\
& \operatorname{Pr}(0111 \mid 1100)=f_{00}\left((1+2 \mu) T_{s}\right) \bar{g}_{00}\left((1+2 \mu) T_{s}\right) ; \\
& \operatorname{Pr}(1000 \mid 0000)=\operatorname{Pr}(1001 \mid 0100)=\operatorname{Pr}(1010 \mid 1000) \\
& \quad=\bar{f}_{00}\left(2 \mu T_{s}\right) g_{00}\left(2 \mu T_{s}\right), \\
& \operatorname{Pr}(1011 \mid 1100)=\bar{f}_{00}\left((1+2 \mu) T_{s}\right) g_{00}\left((1+2 \mu) T_{s}\right) ; \\
& \operatorname{Pr}(1100 \mid 0000)=\operatorname{Pr}(1101 \mid 0100)=\operatorname{Pr}(1110 \mid 1000) \\
& \quad=\bar{f}_{00}\left(2 \mu T_{s}\right) \bar{g}_{00}\left(2 \mu T_{s}\right), \\
& \operatorname{Pr}(1111 \mid 1100)=\bar{f}_{00}\left((1+2 \mu) T_{s}\right) g_{00}\left((1+2 \mu) T_{s}\right) .
\end{aligned}
$$

The stationary distribution $\left(\pi_{x_{1} x_{2}, y_{1} y_{2}}\right)$ can thus be computed numerically. Denoting $\pi_{11}:=\pi_{1110}+\pi_{1101}+\pi_{1100}+$ $\pi_{1111}$, we know that the information rate achieved is

$$
\begin{aligned}
& \frac{\pi_{11} \cdot e^{-\lambda\left(1-e^{-\left|h_{0}\right|^{2}}\right) T_{s}} e^{-\lambda_{1} T_{s}} \cdot W \log \left(1+h_{0}^{2} P\right) T_{s}}{\pi_{11}(1+\mu) T_{s}+\left(1-\pi_{11}\right) \mu T_{s}} \\
= & \frac{\pi_{11} \cdot e^{-\lambda\left(1-e^{-\left|h_{0}\right|^{2}}\right) T_{s}} e^{-\lambda_{1} T_{s}} \cdot W \log \left(1+\left|h_{0}\right|^{2} P\right)}{\pi_{11}(1+\mu)+\left(1-\pi_{11}\right) \mu} .
\end{aligned}
$$

In this policy, finding the best coding threshold $\left|h_{0}\right|^{2}$ becomes complicated because the parameters of both Net2 and Net1 interact deeply in the scheduling.

\section{NETWORK CODING WITH SCHEDUL-} ING

In the previous section, when there is a transmission from Net1, only the intended node is receiving the packet. In this section, we consider a simple network coding where a packet can be received by two nodes. Given that Net1 can receive acknowledgement from the nodes, it can utilize this information to send more than one packet in one transmission if there is such chance.

The scheme works as follows. Net1 detects both nodes at the same time, using $T_{p}$ seconds. If only one node is good such that it is idle in Net2 and also has a good physical channel, then Net1 transmits only to this node for $T_{s}$ seconds. If neither node is good, then Net1 keeps detecting. If both nodes are good, then Net1 uses the following $T_{s}$ seconds as a network coding slot. Net1 takes the following procedure as described in [11] [13] in these network coding slots: 
- STEP 0 (Initialization): Net1 chooses a node from $\{A, B\}$ uniformly as Destination.

Set $N C$ counter $=0$.

- STEP 1: Net1 notifies both nodes Destination, and broadcasts a data packet intended for Destination. If Destination receives it successively or neither node receives correctly, go to STEP 1; if otherwise

(Destination does not receive but the other receiver does), the node who receives correctly put this packet PKT(Destination) in memory, and:

- If NCcounter $=0$, set NCcounter $=1$, switch Destination, and go to STEP 1;

- If NCcounter $=1$, go to STEP 2 .

- STEP 2: Notify both nodes that the next transmission is to broadcasts $P K T(A) \oplus P K T(B)$.

- If neither receiver receives correctly, go to STEP 2 ;

- If both receive correctly, node A decodes $P K T(A)$ by XOR-ing with the received packet with $P K T(B)$, and similarly node B decodes $P K T(B)$ by XOR-ing with the received packet with $P K T(A)$. Go to STEP 0;

- If only A receives, A decodes $P K T(A)$ by XORing with the received packet with $\operatorname{PKT}(B)$. Set Destination $=B$, and go to STEP 1 ;

- If only B receives, B decodes $P K T(B)$ by XORing with the received packet with $P K T(A)$. Set Destination $=A$, and go to STEP 1 ;

For each network coding slot, the probability for one node to fail receiving the packet is

$$
\epsilon:=1-e^{-\lambda\left(1-e^{-\left|h_{0}\right|^{2}}\right) T_{s}} e^{-\lambda_{1} T_{s}},
$$

and the packet has $W \log \left(1+\left|h_{0}\right|^{2} P\right) T_{s}$ bits information. According to [13], the achieved information bits per such network coding slot is

$$
\frac{2\left(1-\epsilon^{2}\right)}{2+\epsilon} W \log \left(1+\left|h_{0}\right|^{2} P\right) T_{s}=: R_{n c} \cdot T_{s} .
$$

In order to calculate the overall performance, the frequency of the different slots - transmitting to only one node, to no node, or to both using network coding - needs to be determined. One can construct a Markov chain as follows. Let $\left(A_{1} A_{2} ; B_{1} B_{2}\right)$ denotes the status of both nodes after each probing, where $A_{1}$ denotes the status of Node1's channel in Net1 and $A_{2}$ the status of Node1's status in Net2. Similarly $\left(B_{1} B_{2}\right)$ denotes the status of Node2. This gives a Markov chain with 16 states. Its transition probability matrix can be determined easily given the transition functions $f_{00}(t), f_{11}(t), g_{00}(t)$ and $g_{11}(t)$ as determined in previous section.

Suppose the stationary distribution is $\left(\pi_{a_{1} a_{2} b_{1} b_{2}}\right)$, and denote $\tilde{\pi}_{11}:=\pi_{1100}+\pi_{1101}+\pi_{1110}$. Then the overall achieved rate is

$$
\frac{\tilde{\pi}_{11} e^{-\lambda\left(1-e^{-\left|h_{0}\right|^{2}}\right) T_{s}} e^{-\lambda_{1} T_{s}} W_{0} T_{s}+\pi_{1111} R_{n c} T_{s}}{\left(\pi_{1111}+\tilde{\pi}_{11}\right)(1+\mu) T_{s}+\left(1-\pi_{1111}-\tilde{\pi}_{11}\right) \mu T_{s}}
$$

$=\frac{\tilde{\pi}_{11} e^{-\lambda\left(1-e^{-\left|h_{0}\right|^{2}}\right) T_{s}} e^{-\lambda_{1} T_{s}} W \log \left(1+\left|h_{0}\right|^{2} P\right)+\pi_{1111} R_{n c}}{\left(\pi_{1111}+\tilde{\pi}_{11}\right)(1+\mu)+\left(1-\pi_{1111}-\tilde{\pi}_{11}\right) \mu}$.

\section{CODING ACROSS SLOTS WITH AGGRE- GATE FEEDBACK}

In previous sections, we assume that during the $T_{s}$ seconds that any interruption from Net2 or any bad channel from Net1 will fail the packet. If delay requirement can be relaxed, Net1 can send multiple slots to a node continuously and only requires one feedback at the end. This way saves time for probing and acknowledgement. We also consider the case when each node is serviced by Net1 with long time and with small $T_{s}$ and the case when probing and ACK need zero time.

\subsection{Transmit Multiple Slots with Aggregate Feedback}

In this case we assume that Net1 detects each user sequentially. If a user is available from Net2 after the detection $\left(T_{p}\right)$, then Net1 starts transmitting $L$ packets to the node, each packet lasts $T_{s}$ seconds. After this batch, the node will feedback which packets are received. If one of the $T_{s}$ 's is interrupted by Net2, then the corresponding packet is considered lost. The feedback needs $T_{a}$ second, and this will let Net1 know which packets are received and which are not. If a user is not available in Net1 after the detection, then Net1 turns to the next node for detection.

Now we calculate the performance. The key is to calculate the average number of packets of the $L$ packets which are received successfully. For $l$-th packet, $1 \leq l \leq L$, the node needs to be available from Net2 at the start $l T_{s}$, and Net2 will not interrupt during the next $T_{s}$ seconds (The conditional probability is $e^{-\lambda_{1} T_{s}}$ ). So the probability that packet $l$ is a success is $h_{11}\left(l T_{s}\right) e^{-\lambda_{1} T_{s}}$ when the PHY channel is not considered. For a successful transmission, the PHY channel must be good too. This has probability $p_{0} e^{-\lambda\left(1-p_{0}\right) T_{s}}$ because the fading process is stationary and is independent of Net2.

In sum, the average number of packets received successfully during the $L T_{s}$ seconds is

$$
\begin{aligned}
& \sum_{l=1}^{L} h_{11}\left(l T_{s}\right) e^{-\lambda_{1} T_{s}} p_{0} e^{-\lambda\left(1-p_{0}\right) T_{s}} \\
& =\sum_{l=1}^{L}\left(\frac{\lambda_{1}}{\lambda_{1}+\lambda_{2}} e^{-\left(\lambda_{1}+\lambda_{2}\right) l T_{s}}+\frac{\lambda_{2}}{\lambda_{1}+\lambda_{2}}\right) \\
& =\left(\frac{\lambda_{1}}{\lambda_{1}+\lambda_{2}} \frac{1-e^{-\lambda_{1} T_{s}} p_{0} e^{\lambda\left(1-p_{0}\right) T_{s}}}{1-e^{\left.-\left(\lambda_{1}+\lambda_{2}\right) T_{s}\right) T_{s}}}+\frac{\lambda_{2} L}{\lambda_{1}+\lambda_{2}}\right) \\
& e^{-\lambda_{1} T_{s}} p_{0} e^{\lambda\left(1-p_{0}\right) T_{s}} .
\end{aligned}
$$

So the average throughput is

$$
\begin{gathered}
\frac{\left(\pi_{10}+\pi_{11}\right)\left(\frac{\lambda_{1}}{\lambda_{1}+\lambda_{2}} \frac{1-e^{-L\left(\lambda_{1}+\lambda_{2}\right) T_{s}}}{1-e^{-\left(\lambda_{1}+\lambda_{2}\right) T_{s}}}+\frac{\lambda_{2} L}{\lambda_{1}+\lambda_{2}}\right)}{\left(\pi_{10}+\pi_{11}\right)(L+\mu)+\left(\pi_{00}+\pi_{01}\right) \mu} \\
\cdot e^{-\lambda_{1} T_{s}} p_{0} e^{\lambda\left(1-p_{0}\right) T_{s}} W \log \left(1+\left|h_{0}\right|^{2} P\right)
\end{gathered}
$$


where $\left(\pi_{i j}\right)$ is the stationary distribution of the Markov chain as in Section 3.2 (by replacing $T_{s}$ with $L T_{s}$ ).

REMARK 1. We can also consider the scheduling where Net1 only transmits to a node when it is both available from Net2 and with a good channel in Net1. This can be solved by constructing a Markov chain and solving it numerically. It is easy to see that when extremely small $T_{s}$ and large $L$ (with $L T_{s}$ being large) are assumed, this scheduling achieves similar performance as what we considered here.

\subsection{The Extreme Case When $T_{s}$ Is Small and $L T_{s}$ Is Large}

If one lets $T_{s} \rightarrow 0$ and $L T_{s} \rightarrow \infty$ in the above policy, it becomes equivalent to the following coding and scheduling. Net1 transmits to the two nodes alternatively for $\bar{T}_{s}$ seconds, and any bit received during the period with the channel being above the coding threshold is considered received correctly. Here $\bar{T}_{s}$ is large, and is equivalently to $L T_{s}$ in previous subsection.

Because both the Net1 channel and Net2 are stationary, the fraction of time when Node1 has good channel and idle from Net2 equals the probability that Node1 has good channel and idle from Net2 in stationary distribution. The probability is $\frac{\lambda_{2}}{\lambda_{1}+\lambda_{2}} \cdot e^{-\left|h_{0}\right|^{2}}$. So the overall information throughput is

$$
\frac{\lambda_{2}}{\lambda_{1}+\lambda_{2}} \cdot e^{-\left|h_{0}\right|^{2}} W \log \left(1+\left|h_{0}\right|^{2} P\right) .
$$

This can also be verified by taking $T_{s} \rightarrow 0$ and $L T_{s} \rightarrow \infty$ in (2).

\subsection{The Extreme Case When Both $\mu$ and $T_{s}$ Is Small}

If both the probing and ACK time $T_{p}+T_{a}$ and transmission time $T_{s}$ are small and $\mu$ goes to zero, then Net1 can successfully transmit whenever there is a node available from Net2 and with a good channel. There is no need for network coding. ${ }^{1}$

In this case, the overall rate achieved is

$$
\left(1-\left(1-\frac{\lambda_{2}}{\lambda_{1}+\lambda_{2}} e^{-\left|h_{0}\right|^{2}}\right)^{2}\right) W \log \left(1+\left|h_{0}\right|^{2} P\right) .
$$

\section{NUMERICAL RESULTS}

In this section, we plot and discuss the performances of the aforementioned strategies.

To illustrate the performance, fix $P=15 \mathrm{~dB}, \mathrm{~W}=1 \mathrm{MHz}$, $T_{s}=1 \mathrm{~ms}, \mu=0.1$. Assume that the average service time in Net2 is $5 \mathrm{~ms}$ (i.e., $1 / \lambda_{2}=5 \mathrm{~ms}$ ) and in Net1 is $1 \mathrm{~ms}$. To see how the activity in Net2 influences the performance, we vary $\lambda_{1}$. As shown in Figure 2, if Net2 is not busy (with a large $1 / \lambda_{1}$ ), then network coding scheme outperforms all other scheduling except the extreme case when Net1 detects

${ }^{1}$ We simplify the decoding by assuming that decoding is always successful whenever the channel is above the coding threshold. However one should note that the decoding is always subject to thermal noise in reality. with zero time and coding is zero length. However the relative gain of network coding diminishes as the Net2 idle time increases. Another observation is that detect and feedback achieve much better performance than even the best time sharing - the red star line. When Net2 is busy with a small average idle time $\left(1 / \lambda_{1}\right)$, as shown in Figure 3, the interruption from Net2 becomes dominant and kills the performance. Network coding's performance shrinks to just that of detect and transmit. The idealized coding achieves much better relative gains but needs fast detection with infinitely small coding length, which is limited by practical considerations. One should caution here that the coding is assumed to be simple such that the ratio $\left|h_{0}\right|^{2}$ plays an important rule in decoding. Coding with advanced features such as waterfilling across channel realizations is not discussed. Also, for simplicity, $\left|h_{0}\right|^{2}$ is the same for all the strategies.

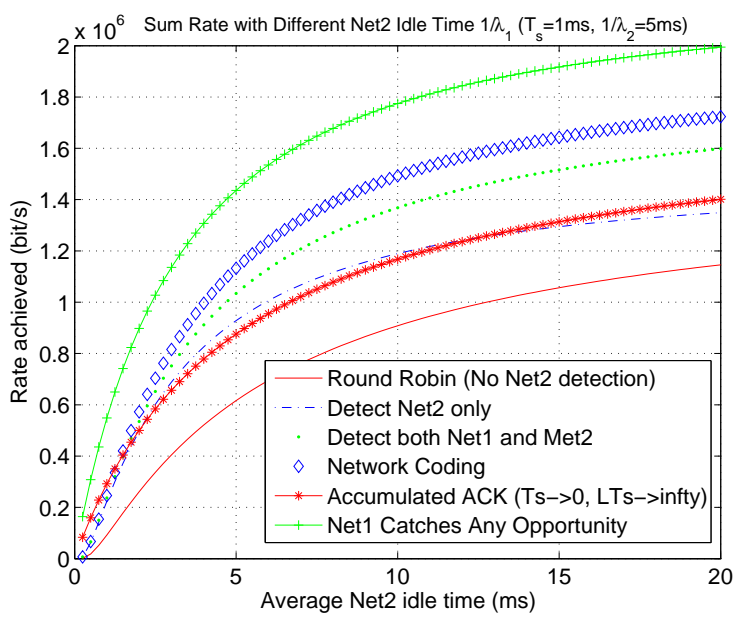

Figure 2: Performance when Net2 has long idle time.

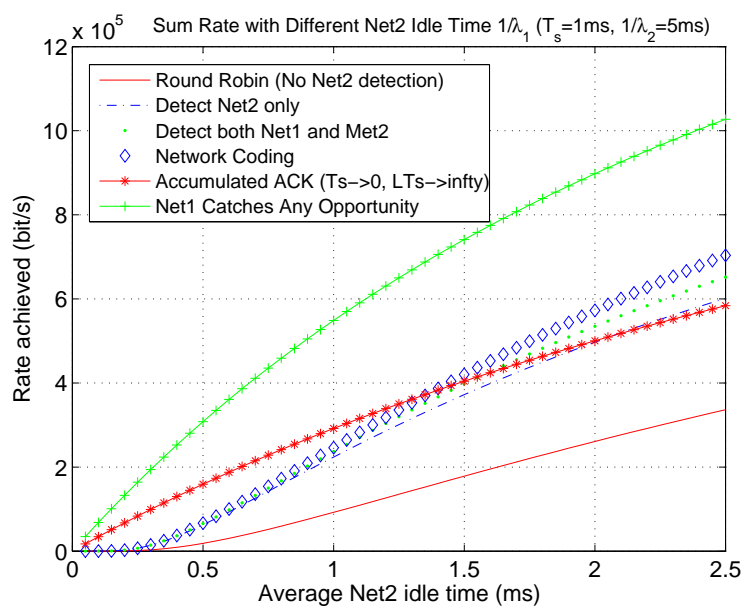

Figure 3: Performance when Net2 is busy.

Figure 4 shows how $T_{s}$ - the Net1 coding length and service 
time - influences the performance with regard to a fixed detection and acknowledgement time $T_{p}+T_{a}$. As expected, smaller $T_{s}$ achieves better performance until $T_{p}+T_{a}$ prevents this trend. This is because as $T_{s}$ becomes smaller, the fraction of time spent in detection and acknowledgement becomes significant.

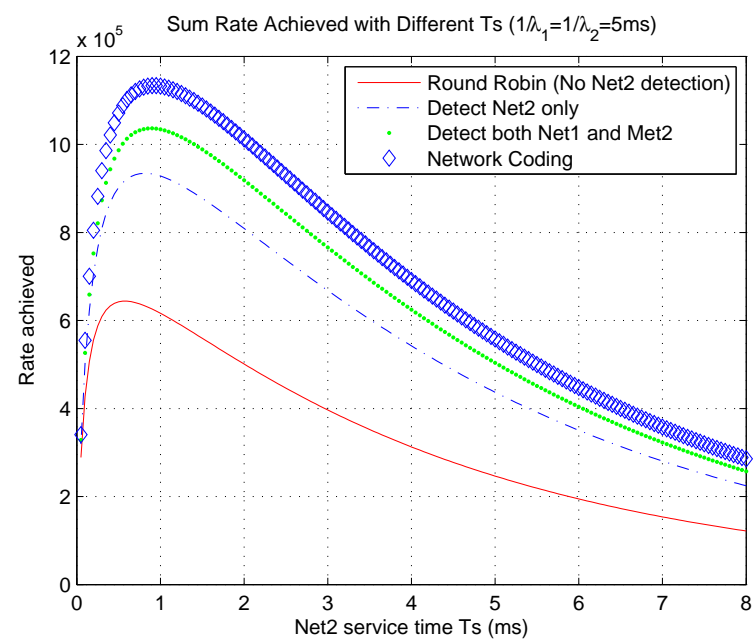

Figure 4: Performance showing that $T_{s}$ is a tradeoff between detection/ACK and coding.

\section{CONCLUDING REMARKS}

This paper discusses a simple coexistence model with two radios (networks) and two nodes. Performance of several scheduling and coding strategies are considered. It shows that in such multi-radio environment, joint coding and scheduling is needed for achieving better throughput. Network coding and accumulated acknowledgement (one example is fountain code and HARQ) could achieve better gain by fighting the frequent interruption. From a more practical point of view, the extra overhead of such coding (memory, sequencing, etc.) needs also be considered. Another observation is that it is hard to characterize the performance of varies strategies by analyzing the corresponding Markov chain. As the number of nodes grows large, the state space grows much faster. A future work is to simplify the modeling with smaller state space and to generalize strategies to larger networks with more nodes and more radios.

\section{REFERENCES}

[1] WPAN/WLAN/WWAN Multi-Radio Coexistence, IEEE 802 Plenary, Atlanta Tuesday, November 13, 2007.

[2] J. Zhu, A. Waltho, Y. Xue and X. Guo, " Multi-radio coexistence: Challenges and opportunities," in Proceedings of 16th International Conference on Computer Communications and Networks (ICCCN 2007), pp. 359-364, 13-16 Aug. 2007.

[3] M. Mittelbach, Muller, C. Ferger, D. Finger, "Study of coexistence between UWB and narrowband cellular systems," in International Workshop on Ultra Wideband Systems, Conference on Ultrawideband Systems and Technologies 2004, pp. 40-44, 18-21, May 2004 .
[4] J. Bellorado, S.S. Ghassemzadeh, L.J. Greenstein, T. Sveinsson and V. Tarokh, "Coexistence of ultra-wideband systems with IEEE-802.11a wireless LANs", in Globecom 2003.

[5] A. Adya, P. Bahl, J. Padhye, A. Wolman and L. Zhou, "A multi-radio unification protocol for IEEE 802.11 wireless networks," in Proceedings of First International Conference on Broadband Networks, 2004. pp. 344-354, 25-29 Oct. 2004.

[6] "Cognitive radio: Brain-empowered wireless communications," in IEEE Journal on Selected Areas in Communications, VOL. 23, NO. 2, pp. 201-220, Feb. 2005.

[7] M. Kodialam and T. Nandagopal, "Characterizing the capacity region in multi-radio multi-channel wireless mesh networks," in Proceedings of the 11th annual international conference on Mobile computing and networking, Cologne, Germany, pp. 73-87, 2005.

[8] L. Yang, L. Cao and H. Zheng, "Proactive channel access in dynamic spectrum networks," in Proceedings of Second International Conference on Cognitive Radio Oriented Wireless Networks and Communications (CROWNCOM), Orlando, FL, August 2007.

[9] S. Katti, D. Katabi, H. Balakrishnan and M. Medard, "Symbol-level network coding for wireless mesh networks," in Proc. SIGCOMM 2008.

[10] D. Nguyen, T. Nguyen, X. Yang, "Multimedia wireless transmission with network coding," in Proceedings of Packet Video 2007, pp. 326-335, Nov. 2007.

[11] Y.E. Sagduyu and A. Ephremides, "On broadcast stability region in random access through network coding," in the 44th Annual Allerton Conference on Communication, Control and Computing, Sep. 2006.

[12] F. Xue, C.-H. Liu and S. Sandhu, "MAC-Layer and PHY-layer network coding for two-way relaying: achievable rate regions and opportunistic scheduling," in the 45th Annual Allerton Conference on Communication, Control and Computing, Sep. 2007.

[13] F. Xue and X. Yang, "Network coding and packet-erasure broadcast channel," in First IEEE International Workshop on Wireless Network Coding, SECON 2008, San Francisco, 2008.

[14] Y. Liang and V.V. Veeravalli, "Capacity of Noncoherent Time-Selective Block Rayleigh Flat-Fading Channel," ISIT 2002, Lausanne, Switzerland, June 30 -July 5, 2002.

[15] D. Bertsekas and R. Gallager, Data networks, Prentice-Hall, Inc. Upper Saddle River, NJ, USA, 1987.

\section{APPENDIX}

\section{A. PROOF FOR THEOREM 1.}

Denote $f:=\log \left(e^{-\left|h_{0}\right|^{2}} \log \left(1+\left|h_{0}\right|^{2} P\right) e^{-\left(1-e^{-\left|h_{0}\right|^{2}} \lambda\right) T_{s}}\right)=$ $-x-T_{s}+e^{-x} \lambda T_{s}+\log \log (1+x P)$, with $x:=\left|h_{0}\right|^{2}$. We know $f^{\prime}=-1-e^{-x} \lambda T_{s}+\frac{P}{(1+x P) \log (1+x P)}$. It is easy to verify that $f^{\prime}>0$ when $x \rightarrow 0^{+}$and $f^{\prime} \rightarrow-1$ when $x \rightarrow \infty$. So there exists an $x$ maximizing $f$ and satisfying $f^{\prime}(x)=0$.

Now we show that such $x$ is unique. We have

$$
f^{\prime \prime}=e^{-x} \lambda T_{s}-P^{2} \frac{1+\log (1+x P)}{(1+x P)^{2}(\log (1+x P))^{2}} .
$$


It is easy to show that $f^{\prime \prime}<0$ when $x \rightarrow 0^{+}$or $x \rightarrow \infty$. So if $f^{\prime}(x)=0$ has more than one solution, $f^{\prime \prime}(x)=0$ has at least one solution with $f^{\prime} \geq 0$ at this solution. Suppose this solution is $y$. Then we have

$$
f^{\prime \prime}(y)=0=e^{-y} \lambda T_{s}-P^{2} \frac{1+\log (1+y P)}{(1+y P)^{2}(\log (1+y P))^{2}} .
$$

So at this point,

$$
\begin{aligned}
& f^{\prime}(y)=-1-P^{2} \frac{1+\log (1+y P)}{(1+y P)^{2}(\log (1+y P))^{2}}+ \\
& =\frac{P}{(1+x P) \log (1+x P)} \\
& <\frac{-(1+y P)^{2}(\log (1+y P))^{2}-P^{2}(1+\log (1+y P))}{(1+y P)^{2}(\log (1+y P))^{2}}+ \\
& \frac{P(1+y P) \log (1+y P)}{(1+y P)^{2}(\log (1+y P))^{2}} \\
& <0,
\end{aligned}
$$

by Cauchy's inequality. This is a contradiction! q.e.d. 